Editorial

\title{
How can the case report survive in a world of big data?
}

\author{
Erdem Aras Sezgin'1D, Tacettin Ayanoğlu² (D), Deniz Çankaya ${ }^{3}$, Baran Sarıkaya ${ }^{4}$ (D) \\ ${ }^{1}$ Department of Orthopedics and Traumatology, Aksaray University, Faculty of Medicine, Aksaray, Turkey \\ ${ }^{2}$ Department of Orthopedics and Traumatology, Abant İzzet Baysal University, Faculty of Medicine, Bolu, Turkey \\ ${ }^{3}$ Department of Orthopedics and Traumatology, Health Sciences University, Gülhane Training and Research Hospital, Ankara, Turkey \\ ${ }^{4}$ Department of Orthopedics and Traumatology, Health Sciences University, City Hospital, Bilkent, Ankara, Turkey
}

We are delighted to publish the first issue of Joint Diseases and Related Surgery Case Reports (JDRSCR). It is a sister journal to Joint Diseases and Related Surgery (https://jointdrs.org) which is indexed in Science Citation Index-Expanded, PubMed/MEDLINE, PubMed Central, and Scopus. In this editorial, we will present our ideas and vision which inspired us to set off with the creation of this new journal.

Orthopedic research got its share of "big data" of today with an ever-growing digitized data being recorded in specific procedure registries, insurance, and administrative databases. We can now relish the opportunity of using the evidence from registry-nested randomized-controlled trials and meta-analyses or registry studies including vast amount of machinereadable data in benefit of our patients and research. ${ }^{[1,2]}$ Making clinical decisions in the light of

Received: November 28, 2021

Accepted: November 28, 2021

Published online: December 22, 2021

Correspondence: Erdem Aras Sezgin, MD. Aksaray Üniversitesi Tıp Fakültesi, Ortopedi ve Travmatoloji Anabilim Dalı, 68100 Aksaray, Türkiye.

E-mail: sezginmd@hotmail.com

Doi: 10.52312/jdrscr.2022.67529

Citation: Sezgin EA, Ayanoğlu T, Çankaya D, Sarıkaya B. How can the case report survive in a world of big data?. Jt Dis Relat Surg Case Rep 2022;1(1):1-2.

(O2022 All right reserved by the Turkish Joint Diseases Foundation

This is an open access article under the terms of the Creative Commons Attribution-NonCommercial License, which permits use, distribution and reproduction in any medium, provided the original work is properly cited and is not used for commercial purposes (http://creativecommons. org/licenses/by-nc/4.0/).

https://www.casereportsjointdrs.org scientifically rigorous evidence is invaluable; however, their availability should not denounce the value of traditional reports on individual experiences from our peers. Case reports are considered to provide the lowest level of evidence, but they can also be regarded as the first-line of evidence. ${ }^{[3]}$ Case reports have a classical aim of reporting a previously unreported, unique clinical conditions or therapeutic procedures. However, unexpected or rare presentations of known diseases or complications can also be the aim, given presented with an informative discussion that highlights the contribution to the literature and main "take-away" lessons. Experiences of colleagues from all around the world on these conditions, which may seldom be encountered during careers of many physicians, can be the only evidence in absence of clinical trials.

Despite cessation of publishing case reports in various highly ranked orthopedic journals, those who continue to publish case reports experience an increasing interest. ${ }^{[4]}$ This trend has led to inception of many case report exclusive journals. Free from costs of hard copy publication, online publishing gives the case report a foundation to thrive further into the $21^{\text {st }}$ century. However, it is of paramount importance to adhere to a standard while reporting clinical experiences. ${ }^{[5]}$ It is not uncommon to come across reports omitting necessary details of medical history, interventions, complications and outcome which significantly reduces their credibility, influence on future research and potential as a guide for clinical practice. ${ }^{[6,7]}$ Journals are the first authority in line to ensure completeness and transparency for case reports, clearly stating the guidelines for authors and through vigorous peer-review. We start by specifying the length of the manuscript, namely word count, number of figures and references in 
JDRSCR. We expect the authors to write their title and abstract in a manner that expresses the originality and how the case report contributes to medicine. An appropriate informed consent for the publication of the case report must be taken from the patient and/or their legal representatives and be stated in the report. In JDRSCR, to ensure completeness, precision and transparency of published case reports, we endorse authors willing to submit their case reports, as well as all our reviewers to adhere to the CAse REports (CARE) guidelines checklist which was developed in 2013. ${ }^{[8]}$ The manuscript should start with establishing the background of the reported condition, referring to the literature. Authors should distinctly present what the reported case adds to what we already know. Details about the case should be objectively presented in the next section starting with demographic information, symptoms, medical history. Then, whole diagnostic process starting from physical examination and all elements of interventions and outcome evaluation should be presented in the case report section. Discussion section should briefly mention similar cases in the literature and emphasize how current case compares. Authors should always acknowledge the inherent limitations of a case report, and base their rationale for conclusions on these.

Only by strictly adhering to standard sof publishing, the case report can be regarded as high-quality reference and continue to exist in the contemporary research environment. We aim to contribute to the persistence of the case report as JDRSCR, by creating an online, open-access, peer-reviewed database formed by experiences of our colleagues across the globe.

\section{REFERENCES}

1. Leonelli S. Scientific Research and Big Data. In: Edward N. Zalta, editor. The Stanford Encyclopedia of Philosophy (Summer 2020 Edition). 2020. Available from: https://plato. stanford.edu/archives/sum2020/entries/science-big-data/ [Accessed: November 18, 2021]

2. Karanatsios B, Prang KH, Verbunt E, Yeung JM, Kelaher M, Gibbs P. Defining key design elements of registry-based randomised controlled trials: A scoping review. Trials 2020;21:552.

3. Jenicek M. Clinical Case Reporting in Evidence-Based Medicine. Oxford: Butterworth-Heinemann; 1999.

4. Bağatur AE, Yalçınkaya M. How to write a case report? Or... not write at all? Eklem Hastalik Cerrahisi 2014;25:165-7.

5. Atik OŞ. Are all case reports worth publishing? Eklem Hastalik Cerrahisi 2016;27:61.

6. Kaszkin-Bettag M, Hildebrandt W. Case reports on cancer therapies: The urgent need to improve the reporting quality. Glob Adv Health Med 2012;1:8-10.

7. Richason TP, Paulson SM, Lowenstein SR, Heard KJ. Case reports describing treatments in the emergency medicine literature: Missing and misleading information. BMC Emerg Med 2009;9:10.

8. Gagnier JJ, Kienle G, Altman DG, Moher D, Sox H, Riley D; CARE Group*. The CARE guidelines: Consensus-based clinical case reporting guideline development. Glob Adv Health Med 2013;2:38-43. 